\title{
Research Paper: The Effect of Neurofeedback on Improving Executive Functions in Children With Attention Deficit/Hyperactivity Disorder
}

\author{
Narges Moin ${ }^{1} \odot$, ${ }^{*}$ Roghayeh Asadi Gandomani ${ }^{2}$ @ , Mohsen Amiri ${ }^{3}$
}

1. Department of Speech Therapy, School of Rehabilitation Sciences, Iran University of Medical Sciences, Tehran, Iran.

2. Department of Psychology, Faculty of Humanities, University of Bojnord, Bojnord, Iran.

3. Department of Psychology, Faculty of Humanities, University of Zanjan, Zanjan, Iran.

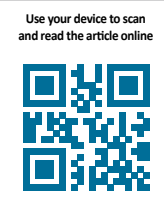

Cfteation Moin N, Asadi Gandomani R, Amiri M. [The Effect of Neurofeedback on Improving Executive Functions in Children With Attention Deficit/Hyperactivity Disorder (Persian)]. Archives of Rehabilitation. 2018; 19(3):220-227. http://dx.doi. org/10.32598/rj.19.3.220

: http://dx.doi.org/10.32598/rj.19.3.220

Keywords:

Attention Deficit/Hy-

peractivity Disorder,

Neurofeedback

Executive functions

\section{ABSTRACT}

Objective Executive functions are processes that control, direct, and coordinate other cognitive processes. The findings of cognitive neuroscience indicate that frontal and temporal lobes have a longer maturity and support the development of executive functions. These abilities are essential for success in everyday activities and longitudinal studies indicate that executive functions are associated with academic achievement, social adaptation, career promotion, and independent performance in children. Executive functions deficits hamper academic achievement and create problematic behaviors. Various studies indicate that children and adolescents with Attention Deficit/Hyperactivity Disorder (ADHD) have a significant disadvantage with respect to the neuropsychological assessment of executive functions (such as planning, inhibition, verbal and spatial memory, and cognitive shifting). ADHD is the most common childhood neurodevelopmental disorder and its symptoms include attention deficit, impulsivity, and hyperactivity. The prevalence of this disorder is higher in boys than in girls. Children with ADHD experience life-long challenges and require support in order to succeed in life; therefore, different intervention approaches have been used to help these children. Recently, neurofeedback has received a lot of attention. It is a type of biofeedback that teaches individual selfregulation by recording electrical responses and providing feedback. The goal of neurofeedback education is to correct abnormal brain wave that improves child's behavioral and cognitive functions. In other words, neurofeedback affects activity of brain waves, so that the brain waves of desirable behaviors are generated or enhanced. Considering the problems that children with ADHD have in executive functions, the present study investigated the effectiveness of neurofeedback on improving executive functions in these children. Materials \& Methods This study was a quasi-experimental research with pretest and post-test. The study population included all children with ADHD in Khorramabad City, Iran. The sample included 10 children with ADHD who were selected by convenience sampling method. The inclusion criteria comprised diagnosis of ADHD confirmed by the psychiatrist and psychologist, normal IQ, and no history of another neurological disease or developmental disorder. In the pretest, the Behavior Rating Inventory of Executive Function (BRIEF) was completed for children. It is one of the most common tools for measuring the executive functions and reflects the teacher's view about student's behavior in the area of executive functions. It screens the executive function problems and covers two areas: metacognition and self-regulation. It measures inhibition, flexibility, emotional control, initiating, working memory, planning-organizing, organization of materials and monitoring. Then, the subjects participated in neurofeedback sessions. Each subject received 10 neurofeedback sessions, twice a week. The beta/theta protocol was administered to all subjects. The purpose of this protocol was to increase beta waves and reduce theta waves. After completing the neurofeedback sessions, BRIEF was completed again in post-test. Descriptive statistics (mean and standard deviation) were used for data analysis and inferential statistics was done by paired t test.

Results The scores of executive functions in the pretest are significantly different from those in the post-test $(P<0.001)$. Also, the findings showed that self-regulation score in the pretest is significantly different from that in the post-test $(\mathrm{P}<0.001)$. In addition, metacognition score of the pretest shows a significant difference from that in the post-test $(P<0.001)$.

Conclusion Neurofeedback can improve the executive functions in children with ADHD. It can help children with ADHD to change their brain waves, and makes them aware of their cognitive performance.

\section{* Corresponding Author:}

Roghayeh Asadi Gandomani, PhD

Address: Department of Psychology, Faculty of Humanities, University of Bojnord, Bojnord, Iran.

Phone: +98 (58) 32201000

E-Mail: psy.assady@gmail.com 


\title{
اثربخشى درمان نوروفيدبك بر بهبود كاركردهاى اجر ايیى در كودكان با اختلال نقص توجه/بيشفعالى
}

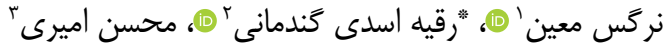

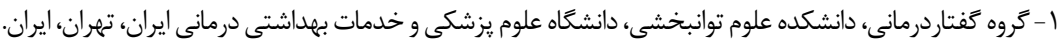

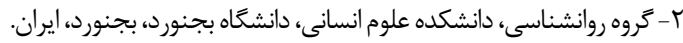

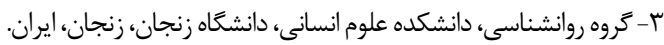

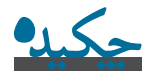

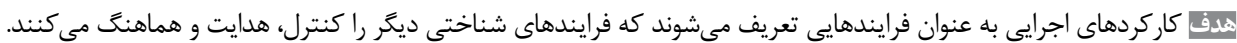

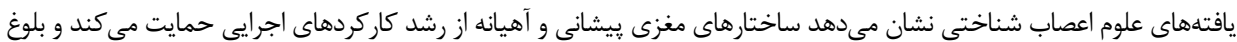

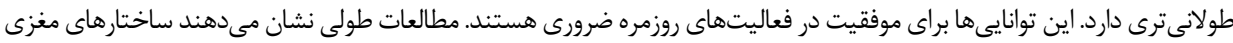

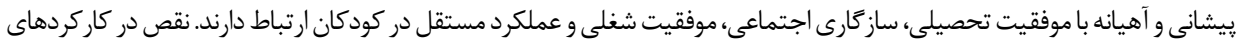

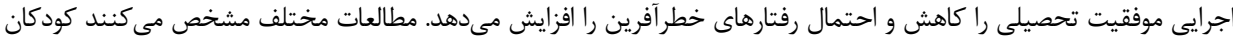

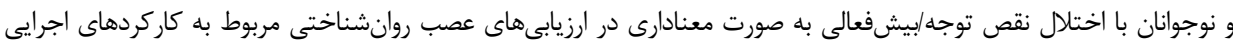

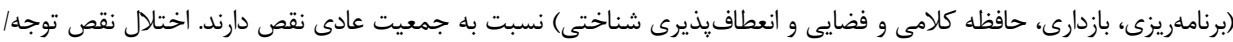

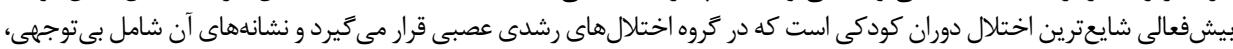

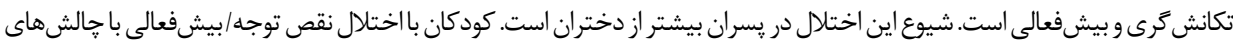

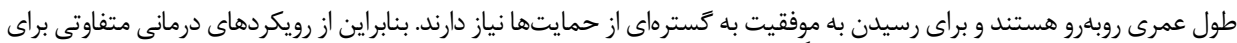

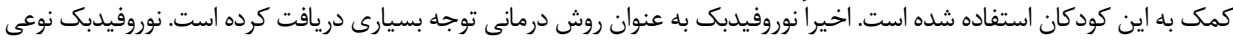

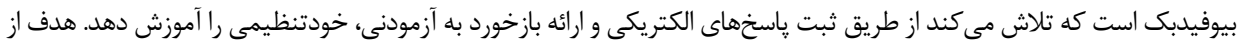

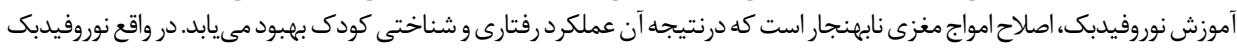

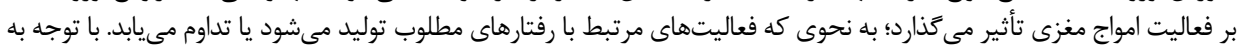

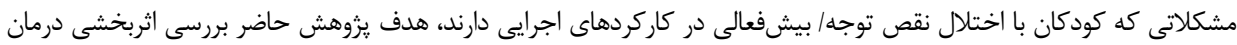

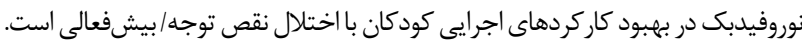

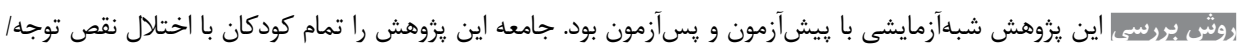

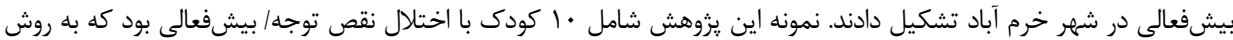

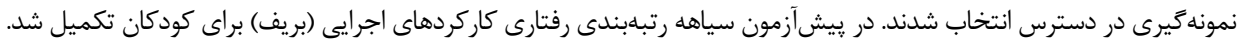

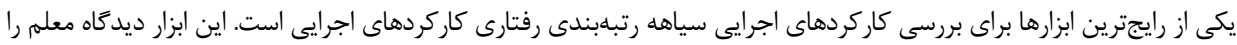

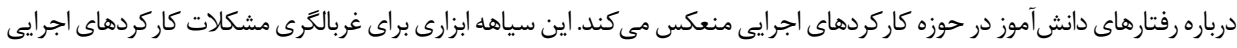

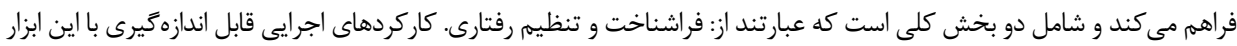

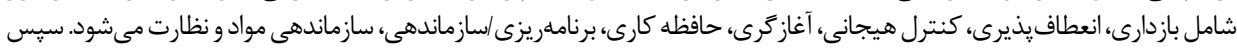

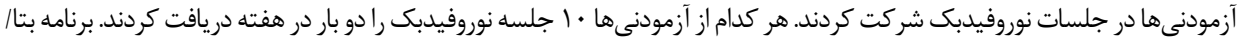

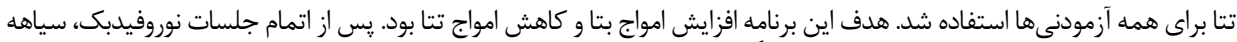

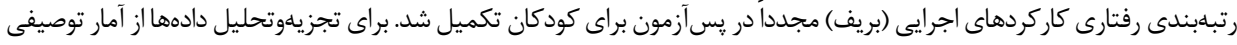

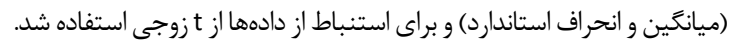

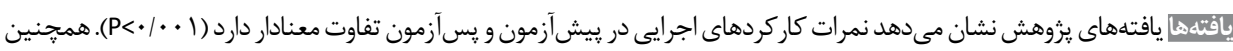

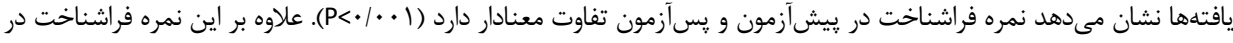

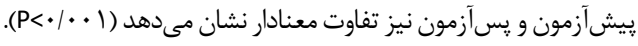

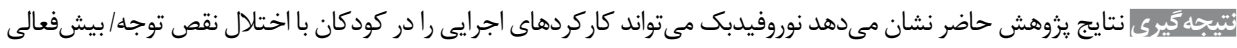

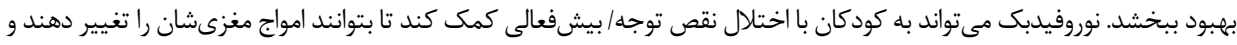

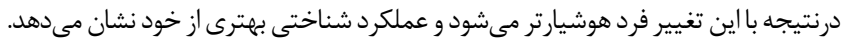

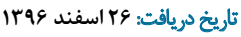 \\ تاريخ هذيرش: 19

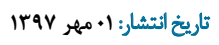

كليدوازوها:

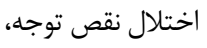

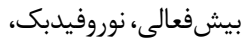
كاركردهاى اجر ايى نوريى

ㄷ․

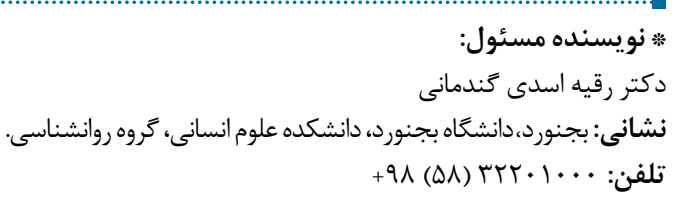

رايانامه: psy.assady@gmail.com 
تركيبى)، f| (ا كودك با اختلال نقص توجهابيشفعالى (از نوع

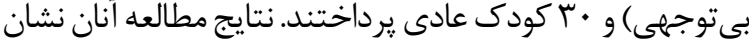

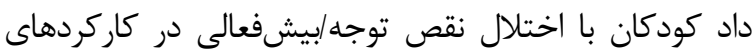

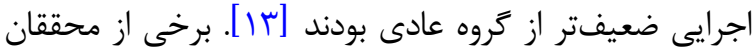

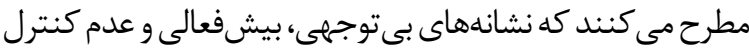

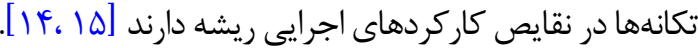

اختلال نقص توجه /بيشفعالى شايعترين اختلال دوران كودكى

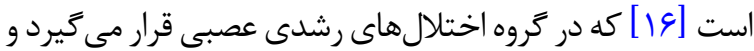

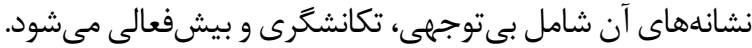

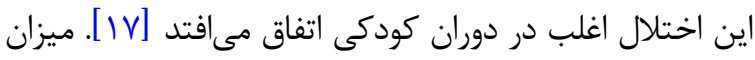

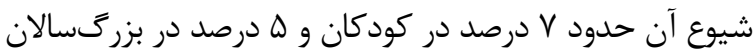

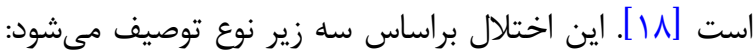

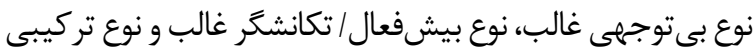

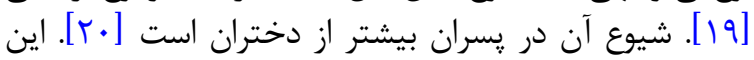

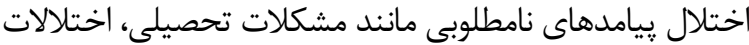

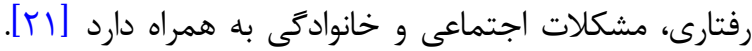

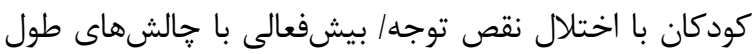

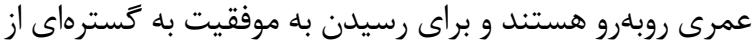

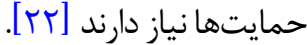

براى كمك به كودكان با اختلال نقص توجه/ بيشفعالى از

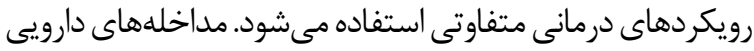

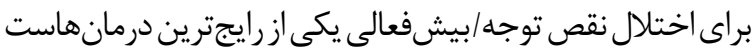

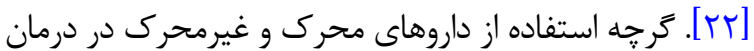

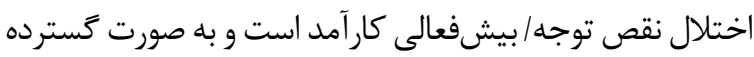

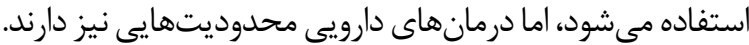

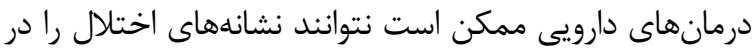

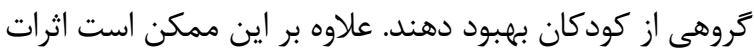

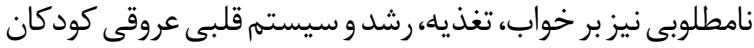

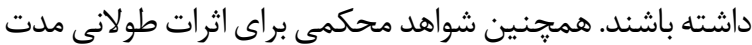

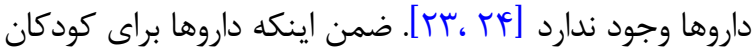

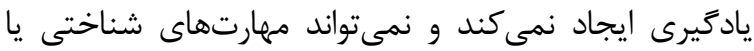

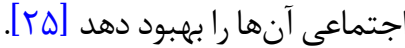

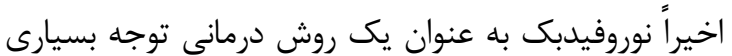

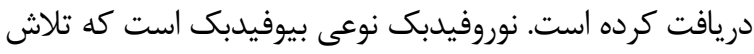

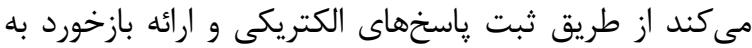

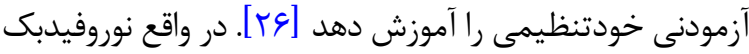

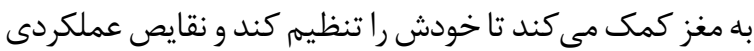

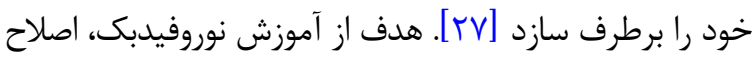

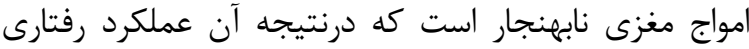

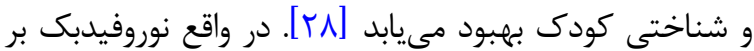

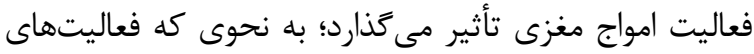

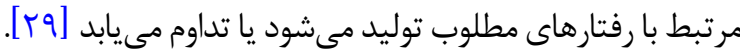

كاركردهاى اجرايى، مهارتهاى كنترل شناختى ناميده

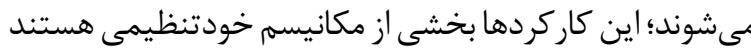

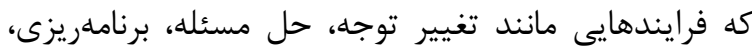

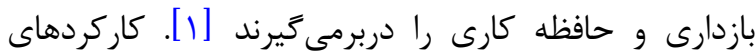

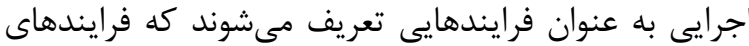

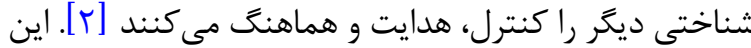

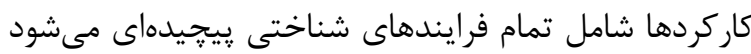

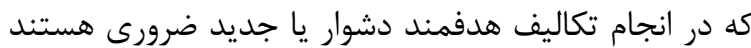

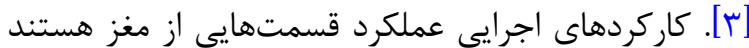

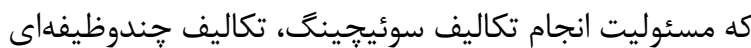

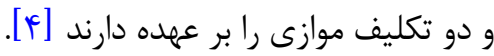
يافتههاى علوم اعصاب شناختى نشان مي دهد ساختارهاى

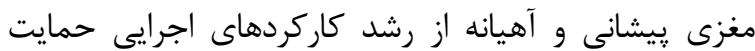

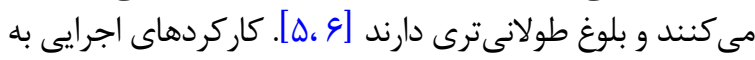

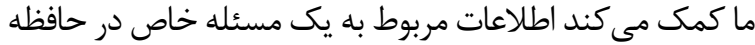

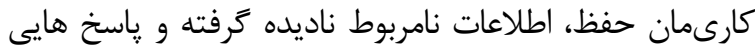

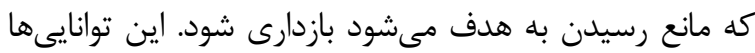

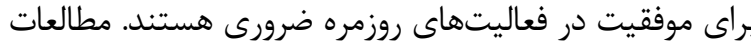

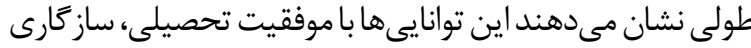
جتماعى و موفقيت شغلى ارتباط دارند [V]

عملكردهاى شناختى را مىتوان به دو بخش سرد و وَرم

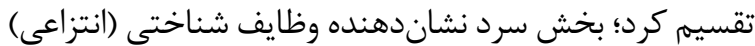

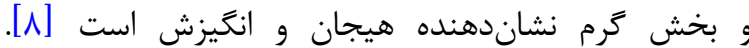

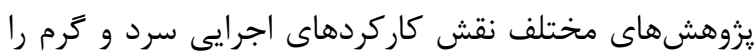

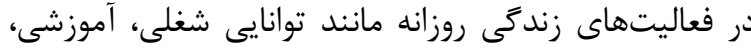

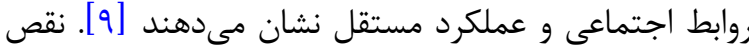

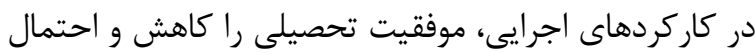

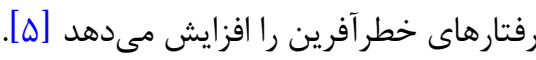
كاركردهاى اجرايى در افراد با اختلالات مختلف آسيب ديده

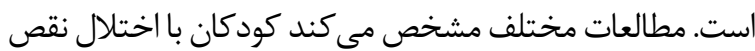

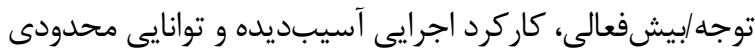

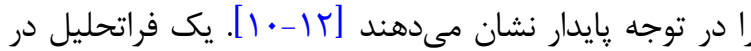

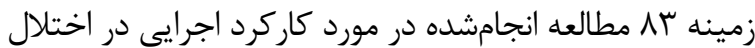

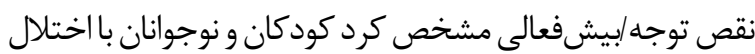

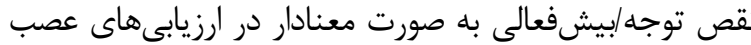

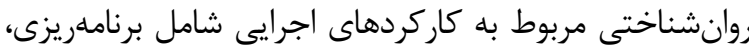

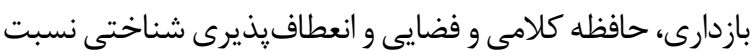

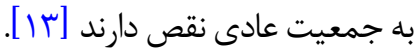
سوريانو فرر و همكاران در يزوهش خود باه بر برسى كاركردهاى

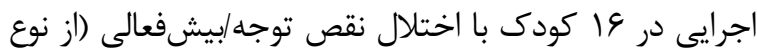




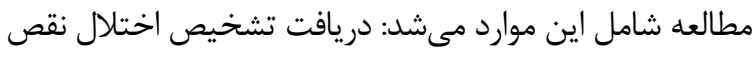

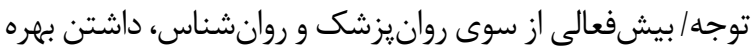

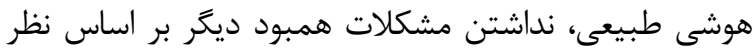

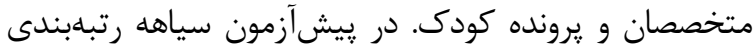

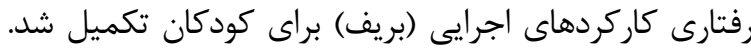

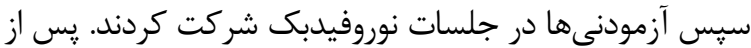

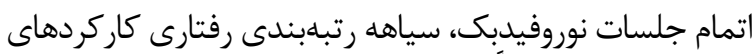

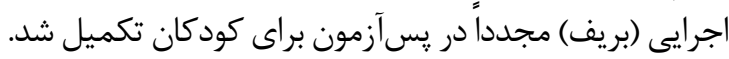

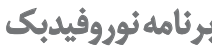

هر كدام از آزمودنىها • ا جلسه نوروفيدبك راد دو بار در هفته

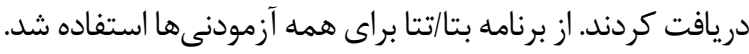

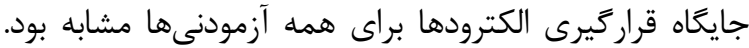

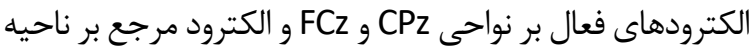

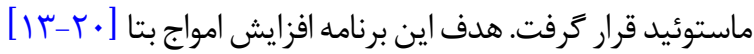

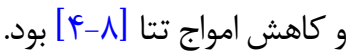

\section{سياهله رتبنبندى رؤتارى كاركردهاى اجرايي (بريف)}

يكى از رايجترين ابزارها براى بررسى كاركردهاى اجرايى،

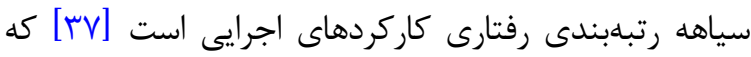

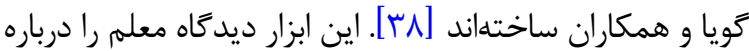

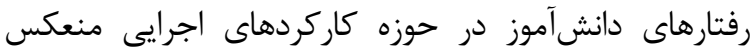

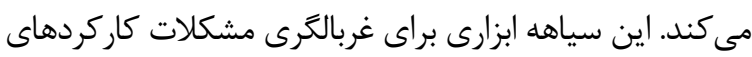

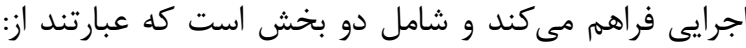

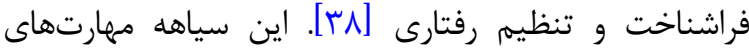

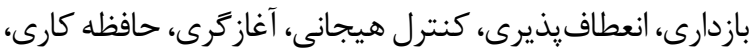

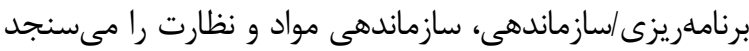

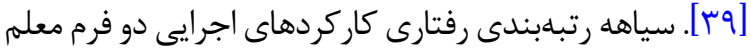

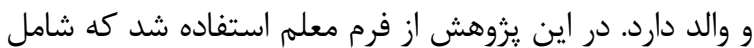

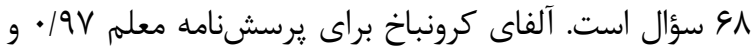

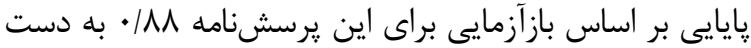

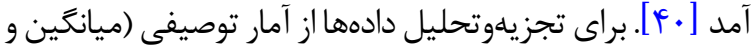
انحر اف استاندارد) و براى استنباط دادهها از t زوجى استفاده شد.

يافتهها

جدول شماره ا نشان مى (مهد دادههاى يزوهش حاضر نرمال

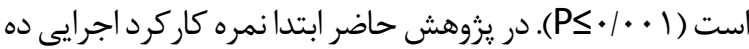

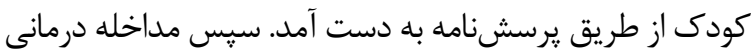

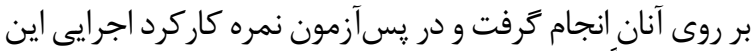

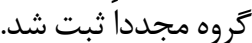

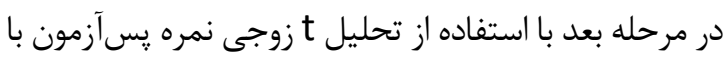

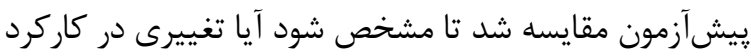
اجرايى يُ از درمان ايجاد شده است يا خير (جدول شماره
فعاليت مغزى در طول نوروفيدبك از طريق الكترودهايى كه بر

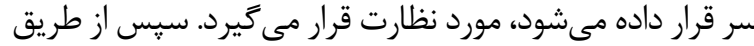

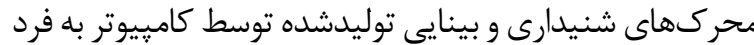

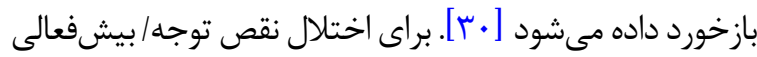

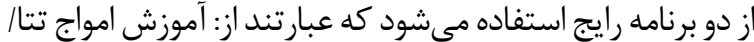

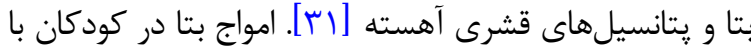

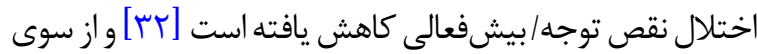

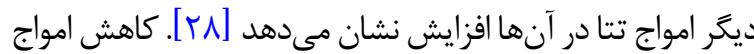

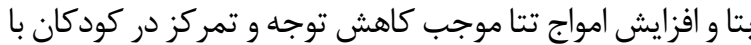

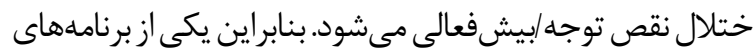

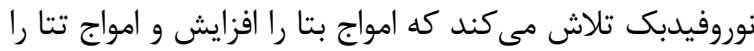

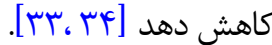

يزوهشهاى بسيارى براى بررسى تأثير نوروفيدبك بر بهبود

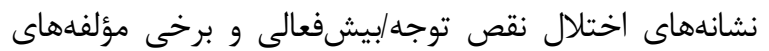

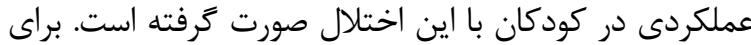

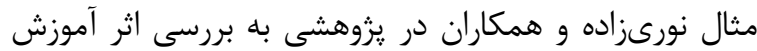

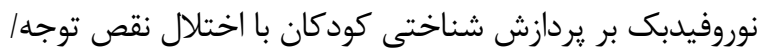

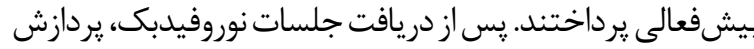

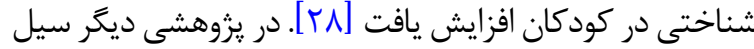

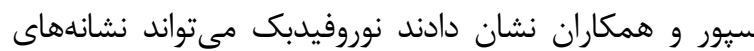

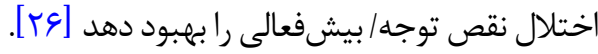

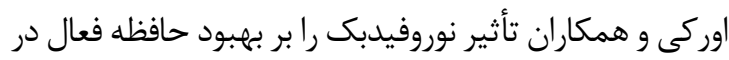

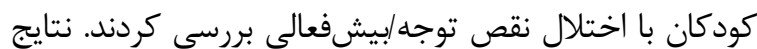

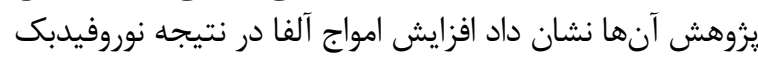

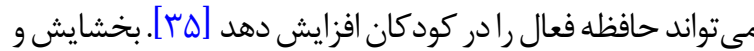

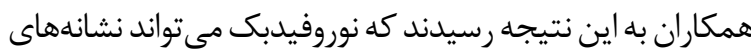

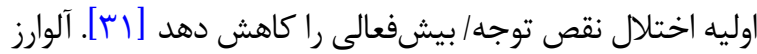

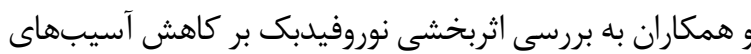

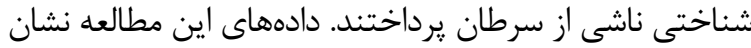

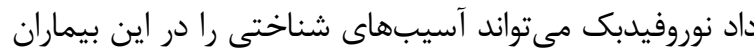

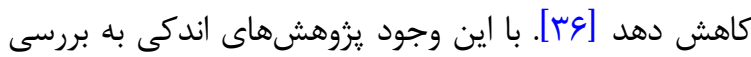

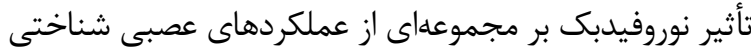

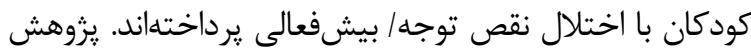

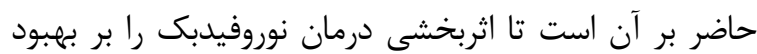

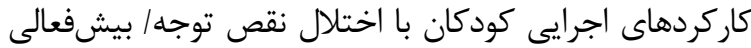

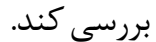

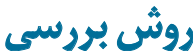

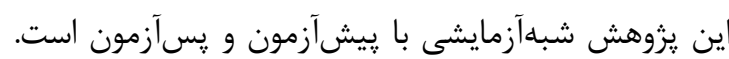

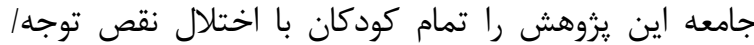

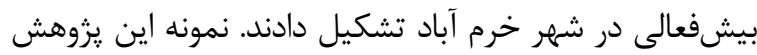

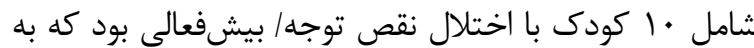

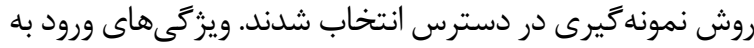


جدول (. نتايج تحليل نرمال بودن دادهها

\begin{tabular}{|c|c|c|c|c|c|c|}
\hline \multicolumn{2}{|c|}{ خودَّردانى } & \multicolumn{2}{|c|}{ فراشناخت } & \multicolumn{2}{|c|}{ كاركرد اجرايى } & \multirow{2}{*}{ آماره } \\
\hline يس"آزمون & ييشآزمون & يسآزمون & ييشآزمون & يسآزمون & يِيشآزمون & \\
\hline.$/ D F$ & . /Va & $.19 \Delta$ & $\cdot / V$ & D & .198 & ks \\
\hline זר/. & . & - /vq & $\cdot / v$. &.$/ 99$ & ./VG & sig. \\
\hline
\end{tabular}

توانبخننى

جدول r. آزمون t زوجى براى بررسى تفاوت بيشآزمون و پسآزمون كاركرد اجرايى

\begin{tabular}{|c|c|c|c|c|c|}
\hline $\mathbf{P}$ & $\mathbf{t}$ & انحراف استاندارد & ميانكين & مرحله & متغير \\
\hline.$/ . .1$ & $\mid r / \cdot r$ & $\begin{array}{l}r \Delta / \Psi T \\
\Gamma / \Delta \Delta\end{array}$ & $\begin{array}{l}M N / r . \\
I K E / \Delta .\end{array}$ & ييش آزمون & كاركرد اجرايى \\
\hline
\end{tabular}

توانبخننى

بازدارى و انعطافيذيرى ذهنى هستند. هدف يزوهش حاضر

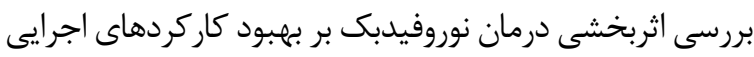

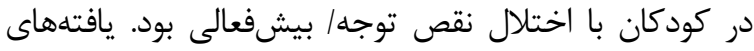

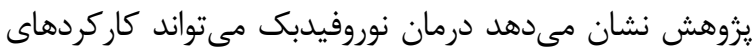

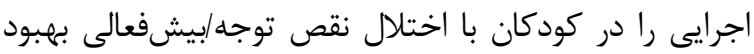

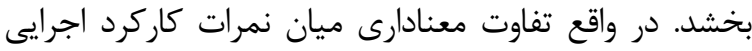

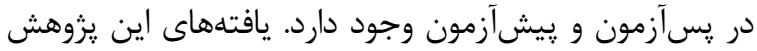

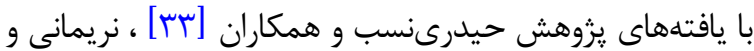

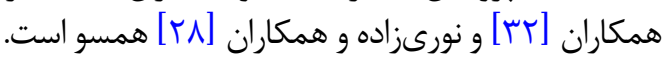

حيدرى نسب و همكاران نشان دادند نوروفيدبك منجر به

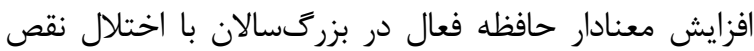

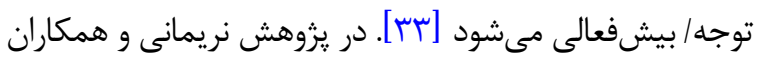

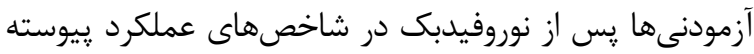

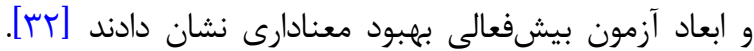

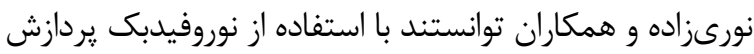

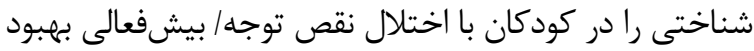

دهند [باخت
Y). همان گونه كه در جدول شماره Y مشاهده مىشود نمره

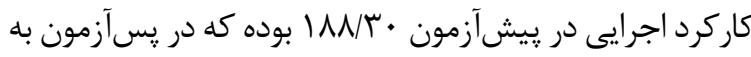

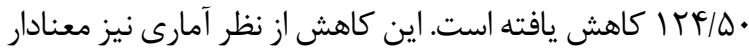

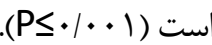

همان گونه كه در جدول شماره r مشاهده مئشود نمره

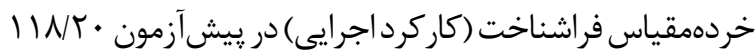

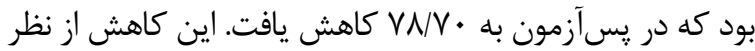

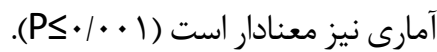

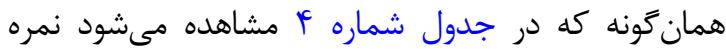

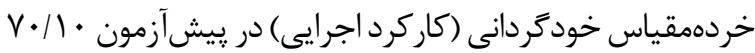

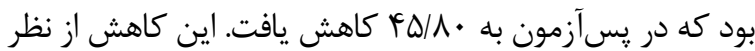

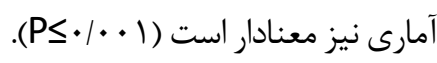

\section{ث}

كاركردهاى اجرايى مجموعهاى از فعاليتهاى شناختى سطح بالا هستند كه براى دستيابى به اهداف مجاف مورد نظر استفاده

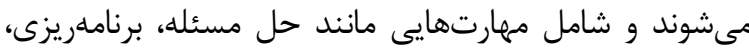

جدول س. آزمون t زوجى براى بررسى تفاوت پيشآزمون و يسآزمون خردهمقياس فراشناخت (كاركرد اجرايى)

\begin{tabular}{|c|c|c|c|c|c|}
\hline $\mathrm{P}$ & $\mathrm{t}$ & انحراف استاندارد & ميانكَين & مرحله & متغير \\
\hline \multirow[b]{2}{*}{ 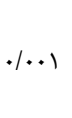 } & \multirow[b]{2}{*}{$1 . / 90$} & $\mid t / 4 F^{4}$ & $\| N / r$. & ييشآزمون & \multirow[b]{2}{*}{ كاركرد اجرايى } \\
\hline & & N/q. & & پِ آزمون & \\
\hline
\end{tabular}

جدول ع. آزمون t زوجى براى بررسى تفاوت يِيشآزمون و پِّآزمون خردهمقياس خودكردانى (كاركرد اجرايى)

\begin{tabular}{|c|c|c|c|c|c|}
\hline$P$ & $\mathrm{t}$ & انحراف استاندارد & ميانكَين & مرحله & متغير \\
\hline \multirow{2}{*}{$.1 . .1$} & \multirow{2}{*}{$V / I V$} & $\mid f / 19$ & $v \cdot / 1$. & بيشآزمون & \multirow{2}{*}{ كاركرد اجرايى } \\
\hline & & $\Delta / \& q$ & $r \Delta / \lambda$. & سֶس آزمون & \\
\hline
\end{tabular}

توانبخنتى 
تحقيق روى نمونه محدودى انجام شد و در تعميم نتايج آن بايد

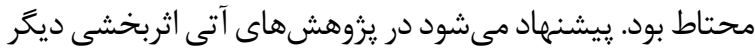

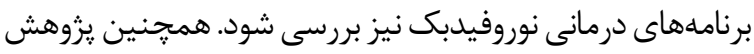
روى نمونه بزرگتر و با مر حله يِيَيرى انجام شود.

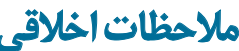
يبيروى از اصول الخلاق يؤهش

همه اصول اخلاقى در اين مقاله رعايت شده است.

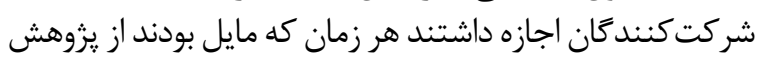

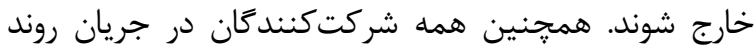

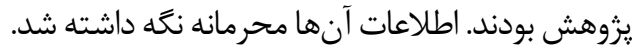

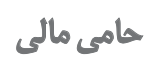

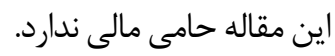

$$
\text { تعارض مئافع }
$$

بنابر اظهار نويسندكان اين مقاله تعارض منافع ندارد.

$$
\text { تشيكر و قبدوراتي }
$$

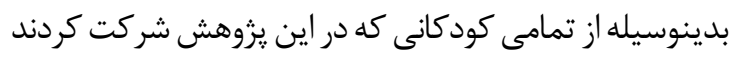

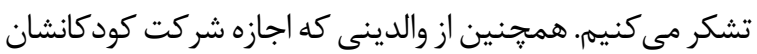

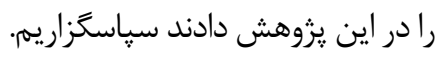

در تبيين يافتههاى حاضر مى توان كفت كه آموزش نوروفيدبك

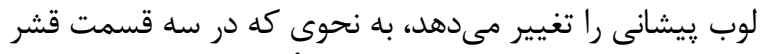

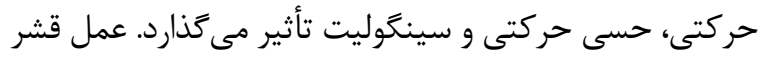

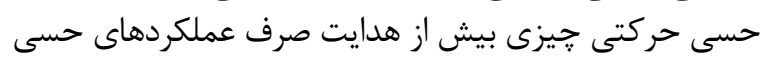

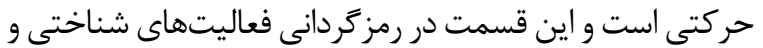

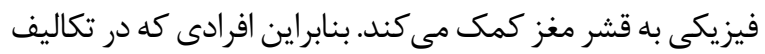

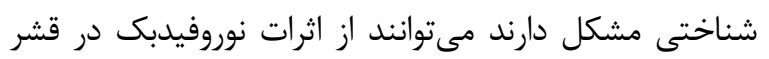

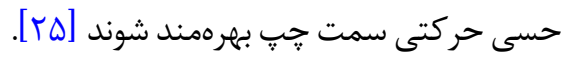

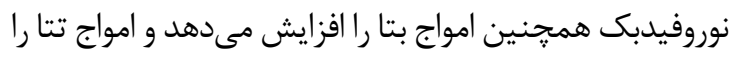

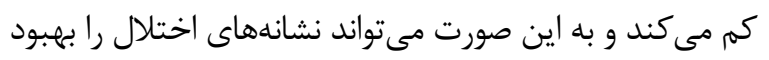

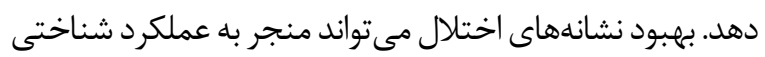

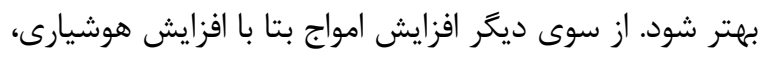

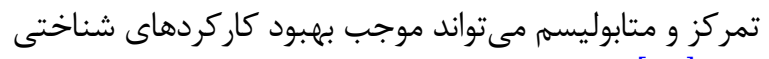

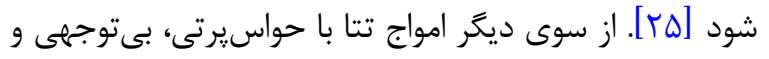

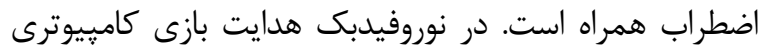

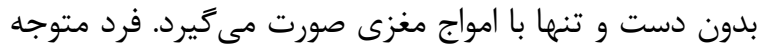

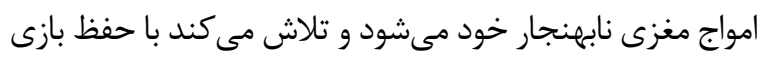

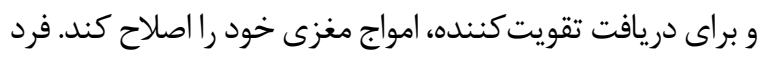

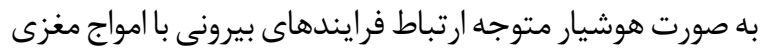

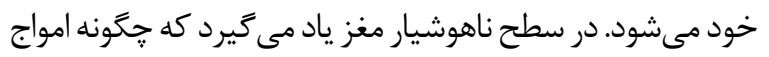

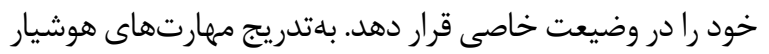

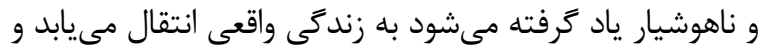
عملكرد فرد را تحت تأثير قرار مى دهند.

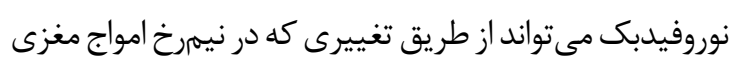

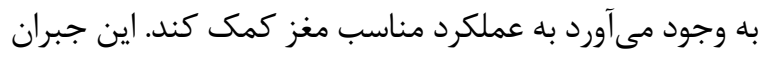

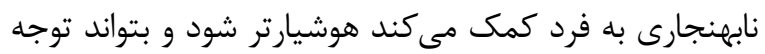

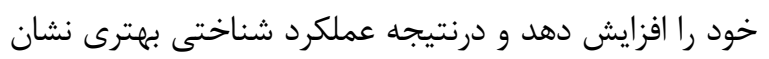

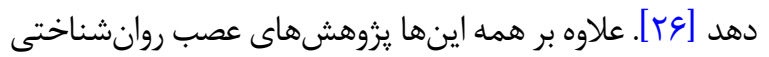

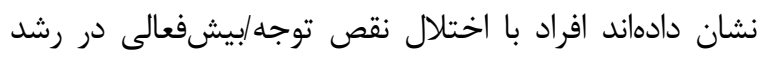

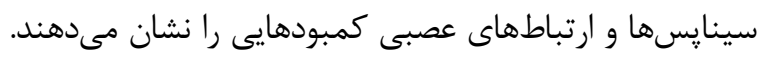

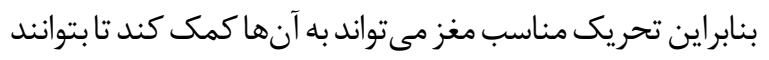

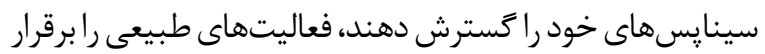

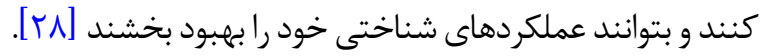

\section{نتيجهَيرى}

نتايج يزوهش حاضر نشان داد نوروفيدبك مى تواند كاركر دهاى

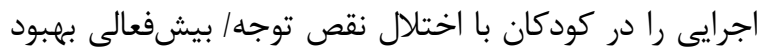

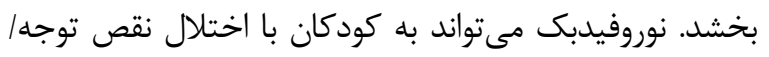

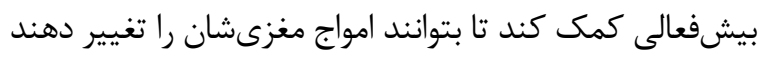

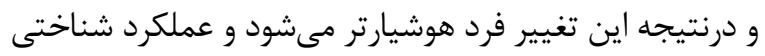
بهترى را نشان مىدهد. اين يزوهش مرحله پييگيرى اثرات درمان راشامل نمىشود. اين 


\section{References}

[1] Sosic-Vasic Z, Kröner J, Schneider S, Vasic N, Spitzer M, Streb $\mathrm{J}$. The association between parenting behavior and executive functioning in children and young adolescents. Frontiers in Psychology. 2017; 8:472. [DOI:10.3389/fpsyg.2017.00472] [PMID] [PMCID]

[2] Bull R, Lee K. Executive functioning and mathematics achievement. Child Development Perspectives. 2014; 8(1):36-41. [DOI:10.1111/cdep.12059]

[3] Amani O, Mazaheri MA, Nejati V, Shamsian BS. [Effect of cognitive rehabilitation on executive functions in adolescent survivors of leukemia: A randomized and controlled clinical trial (Persian)]. Archives of Rehabilitation. 2017; 18(1):73-82. [DOI:10.21859/ jrehab-180173]

[4] Yousefi R, Soleimani M, Ghazanfariyanpoor S. [Comparison between switching and creativity among bilingual and monolingual children (Persian)]. Archives of Rehabilitation. 2017; 18(1):1-12. [DOI:10.21859/jrehab-18011]

[5] Munro BA, Weyandt LL, Marraccini ME, Oster DR. The relationship between nonmedical use of prescription stimulants, executive functioning and academic outcomes. Addictive Behaviors. 2017; 65:250-7. [DOI:10.1016/j.addbeh.2016.08.023] [PMID]

[6] Lee K, Bull R, Ho RM. Developmental changes in executive functioning. Child Development. 2013; 84(6):1933-53. [DOI:10.1111/ cdev.12096] [PMID]

[7] Serpell ZN, Esposito AG. Development of executive functions: implications for educational policy and practice. Policy Insights from the Behavioral and Brain Sciences. 2016; 3(2):203-10. [DOI:10.1177/2372732216654718]

[8] Nejati V. [Correlation of risky decision making with executive function of brain in adolescences (Persian)]. Journal Research Behavioural Science. 2013; 11(4):270-8.

[9] Amani M, Asady Gandomani R, Nesayan A. The reliability and validity of behavior rating inventory of execu-tive functions tool teacher's form among iranian primary school students. Iranian Rehabilitation Journal. 2018; 16(1):25-34. [DOI:10.29252/nrip. irj.16.1.25]

[10] Tye C, Bedford R, Asherson P, Ashwood K, Azadi B, Bolton P, et al. Callous-unemotional traits moderate executive function in children with ASD and ADHD: A pilot event-related potential study. Developmental Cognitive Neuroscience. 2017; 26:84-90. [DOI:10.1016/j.den.2017.06.002] [PMID] [PMCID]

[11] Tehrani Dust M, Radgudarzi R, Sepasi M, Alagheband Rad J. Executive functions in children with attention deficit hyperactivity disorder. Advances in Cognitive Science. 2003; 5(1):1-9.

[12] Rapport MD, Orban SA, Kofler MJ, Friedman LM. Do programs designed to train working memory, other executive functions, and attention benefit children with ADHD? A meta-analytic review of cognitive, academic, and behavioral outcomes. Clinical Psychology Review. 2013; 33(8):1237-52. [DOI:10.1016/j. cpr.2013.08.005] [PMID]

[13] Soriano-Ferrer M, Félix-Mateo V, Begeny J. Executive function domains among children with ADHD: Do they differ between parents and teachers ratings. Procedia-Social and Behavioral Sciences. 2014; 132:80-6. [DOI:10.1016/j.sbspro.2014.04.281]

[14] Pennington BF, Ozonoff S. Executive functions and developmental psychopathology. Journal of Child Psychology and Psychiatry. 1996; 37(1):51-87. [DOI:10.1111/j.1469-7610.1996. tb01380.x]

[15] Barkley RA. Behavioral inhibition, sustained attention, and executive functions: Constructing a unifying theory of ADHD Psychological Bulletin. 1997; 121(1):65-94. [DOI:10.1037/00332909.121.1.65] [PMID]

[16] Ziereis S, Jansen P. Effects of physical activity on executive function and motor performance in children with ADHD. Research in Developmental Disabilities. 2015; 38:181-91. [DOI:10.1016/i. ridd.2014.12.005] [PMID]

[17] Lee EJ, Jung CH. Additive effects of neurofeedback on the treatment of ADHD: A randomized controlled study. Asian Journal of Psychiatry. 2017; 25:16-21. [DOI:10.1016/j.ajp.2016.09.002] [PMID]

[18] Fried R, Chan J, Feinberg L, Pope A, Woodworth KY, Faraone $\mathrm{SV}$, et al. Clinical correlates of working memory deficits in youth with and without ADHD: A controlled study. Journal of Clinical and Experimental Neuropsychology. 2016; 38(5):487-96. [DOI:1 0.1080/13803395.2015.1127896] [PMID] [PMCID]

[19] Arns M, Heinrich H, Strehl U. Evaluation of neurofeedback in ADHD: The long and winding road. Biological Psychology. 2014, 95:108-15. [DOI:10.1016/j.biopsycho.2013.11.013] [PMID]

[20] Sattari M, Hosseini SA, Rassafiani M, Mahmoudi Gharaei M], Biglarian A, Tarkesh Esfahani N. [Prevalence of comorbidity behavioral disorders in children with attention deficit hyperactivity (Persian)]. Archives of Rehabilitation. 2017; 18(1):25-32. DOI:10.21859/jrehab-180125]

[21] Patros CHG, Alderson RM, Hudec KL, Tarle SJ, Lea SE. Hyperactivity in boys with attention-deficit/hyperactivity disorder: The influence of underlying visuospatial working memory and self-control processes. Journal of Experimental Child Psychology. 2017; 154:1-12. [DOI:10.1016/j.jecp.2016.09.008] [PMID]

[22] Kirk S, Gallagher JJ, Coleman MR, Anastasiow NJ. Educating exceptional children. Boston: Cengage Learning; 2015.

[23] Schönenberg M, Wiedemann E, Schneidt A, Scheeff J, Logemann A, Keune PM, et al. Neurofeedback, sham neurofeedback, and cognitive-behavioural group therapy in adults with attention-deficit hyperactivity disorder: A triple-blind, randomised, controlled trial. The Lancet Psychiatry. 2017; 4(9):673-84 [DOI:10.1016/S2215-0366(17)30291-2]

[24] Holtmann M, Sonuga-Barke E, Cortese S, Brandeis D. Neurofeedback for ADHD: A review of current evidence. Child and Adolescent Psychiatric Clinics of North America. 2014; 23(4):789-806. [DOI:10.1016/j.chc.2014.05.006] [PMID]

[25] Madani A S, Heidarinasab L, Yaghubi H, Rostami R. Surveying Effectiveness of Neuro-feedback in Reduction of Attention and Concentration Deficit Symptoms in ADHD Adults. Clinical Psychology \& Personality. 2014; 2(11):85-98.

[26] Seilsepour M, Hamounpeyma E, Pirkhaefi, A. The effect of Neurofeedback therapy sessions on female elementary students 
with attention deficit and hyperactivity in varamin city, in 2013. Journal of the Student Research Committee. 2013; 18(60): 24-33.

[27] Jahani M, Pishyareh E, Haghgoo HA, Hosseini SA, Ghadamgahi Sani SN. Neurofeedback effect on perceptual-motor skills of children with ADHD. Iranian Rehabilitation Journal. 2016; 14(1):43-50. [DOI:10.15412/J.IRJ.08140107]

[28] Nourizade N, Mikeeli manee F, Rostami R. The effectiveness of neurofeedback training on cognitive processing in children with attention deficit hyperactivity disorder. Journal of School Psychology. 2015; 4(3):119-136.

[29] Hemmati S, Vameghi R, Sajedi F, Gharib M, Pourmohammadreza-Tajrishi M, Teymori R. The effect of neurofeedback on brain waves in children with autism spectrum disorders. Iranian Rehabilitation Journal. 2016; 14(3):133-8. [DOI:10.18869/nrip. irj.14.3.133]

[30] Luctkar-Flude M, Groll D. A systematic review of the safety and effect of Neurofeedback on fatigue and cognition. Integrative Cancer Therapies. 2015; 14(4):318-40. [DOI:10.1177/1534735415572886] [PMID]

[31] Bakhshayesh AR, Hänsch S, Wyschkon A, Rezai MJ, Esser G. Neurofeedback in ADHD: A single-blind randomized controlled trial. European Child \& Adolescent Psychiatry. 2011; 20(9):481. [DOI:10.1007/s00787-011-0208-y] [PMID]

[32] Narimani M, Rajabi S, Delavar S. Effects of neurofeedback training on female students with attention deficit and hyperactivity disorder. Journal of Arak University of Medical Sciences. 2013; 16(2):91-103.

[33] Heidari Nasab L, Madani M, Yaghoobi H, Rostami R, Kazemi R. [Investigating the effectiveness of neurofeedback combined with cognitive computer-based exercises to improve working memory in adults with attention deficit hyperactivity disorder (Persian)]. Yafteh. 2016; 18(1):101-12.

[34] Geladé K, Bink M, Janssen TW, van Mourik R, Maras A, Oosterlaan J. An RCT into the effects of neurofeedback on neurocognitive functioning compared to stimulant medication and physical activity in children with ADHD. European Child \& Adolescent Psychiatry. 2016; 26(4):1-12. [DOI: 10.1007/s00787-016-0902-x] [PMID] [PMCID]

[35] Oraki M, Rahmanian M, Tehrani N, Heidari H. The effect of neuorofeedback instruction on the improvement of the working memory of children with attention deficit and hyperactivity disorder. Journal of Neuropsychology. 2015; 1(1):41-51.

[36] Alvarez J, Meyer FL, Granoff DL, Lundy A. The effect of EEG biofeedback on reducing postcancer cognitive impairment. Integrative Cancer Therapies. 2013; 12(6):475-87. [DOI:10.1177/1534735413477192] [PMID]

[37] Toplak ME, West RF, Stanovich KE. Practitioner Review: Do performance-based measures and ratings of executive function assess the same construct. Journal of Child Psychology and Psychiatry. 2013; 54(2):131-43. [DOI:10.1111/jcpp.12001] [PMID]

[38] Gioia GA, Isquith PK, Guy SC, Kenworthy L. Test review behavior rating inventory of executive function. Child Neuropsychology. 2000; 6(3):235-8.
[39] Anderson B, Storfer-Isser A, Taylor HG, Rosen CL, Redline S. Associations of executive function with sleepiness and sleep duration in adolescents. Pediatrics. 2009; 123(4):701-7. [DOI:10.1542/ peds.2008-1182] [PMID] [PMCID]

[40] Isquith PK, Crawford JS, Espy KA, Gioia GA. Assessment of executive function in preschool-aged children. Developmental Disabilities Research Reviews. 2005; 11(3):209-15. [DOI:10.1002/ mrdd.20075] [PMID] [PMCID] 